\title{
Search of anti-allodynic compounds from Plantaginis Semen, a crude drug ingredient of Kampo formula "Goshajinkigan"
}

\author{
Kazufumi Toume ${ }^{1}$ (D) Zhiyan Hou ${ }^{1} \cdot$ Huanhuan $\mathrm{Yu}^{1} \cdot$ Mitsuru Kato $^{2} \cdot$ Miki Maesaka $^{2}$. Yanjing Bai ${ }^{1} \cdot$ Shiho Hanazawa ${ }^{1}$. \\ Yuewei $\mathrm{Ge}^{1} \cdot$ Tsugunobu Andoh $^{2}$ (1) $\cdot$ Katsuko Komatsu' ${ }^{1}$
}

Received: 21 April 2019 / Accepted: 1 June 2019 / Published online: 12 June 2019

(c) The Author(s) 2019, corrected publication 2020

\begin{abstract}
Chemotherapy-induced peripheral neuropathy (CIPN) is one of the dose-limiting side effects of cancer chemotherapy. Although the control of CIPN is important, it is difficult to manage with currently available therapeutic drugs. Therefore, there is a need for novel therapeutic agents for treating CIPN. Goshajinkigan (GJG) is a Kampo formula composed of ten crude drugs. While GJG has been used for the treatment of CIPN, the active constituents of GJG and their underlying mechanisms of pharmacological effects are still unknown. Our previous study revealed that repetitive oral administration of the water extract of Plantaginis Semen, a crude drug ingredient of GJG, inhibited the mechanical allodynia induced by an intraperitoneal injection of paclitaxel in mice. To elucidate the active compounds of Plantaginis Semen, activity-guided separation of the water extract of Plantaginis Semen was performed. From the active fraction, four iridoids (1-4) were identified. Repetitive oral administration of aucubin (1) at 100 or $30 \mathrm{mg} / \mathrm{kg}$ and $100 \mathrm{mg} / \mathrm{kg}$ of the fraction crude 3 [primarily comprised of pedicularis-lactone (3)], showed anti-allodynic activity, suggesting $\mathbf{1}$ and $\mathbf{3}$ could be some of the active compounds responsible for the anti-allodynic property of Plantaginis Semen and GJG. Our study establishes that oral administration of $\mathbf{1}$ has potent anti-allodynic effect in addition to the activity of intraperitoneally administered $\mathbf{1}$ reported previously. Identification of active anti-allodynic compounds found in Kampo formulations will support the development of novel therapies for the management of CIPN in cancer patients.
\end{abstract}

Keywords Plantaginis Semen $\cdot$ Plantago asiatica $\cdot$ Allodynia $\cdot$ Peripheral neuropathy $\cdot$ Iridoids $\cdot$ Goshajinkigan

\section{Introduction}

Chemotherapy is a widely used form of cancer therapy. Several chemotherapeutic agents including paclitaxel (PTX), oxaliplatin, vincristine, bortezomib, and others cause serious

Electronic supplementary material The online version of this article (https://doi.org/10.1007/s11418-019-01327-2) contains supplementary material, which is available to authorized users.

Kazufumi Toume

toume@inm.u-toyama.ac.jp

$\triangle$ Katsuko Komatsu

katsukok@inm.u-toyama.ac.jp

1 Division of Pharmacognosy, Institute of Natural Medicine, University of Toyama, 2630 Sugitani, Toyama, Toyama 930-0194, Japan

2 Department of Applied Pharmacology, Graduate School of Medicine and Pharmaceutical Sciences, University of Toyama, 2630 Sugitani, Toyama, Toyama 930-0194, Japan side effects such as vomiting and peripheral neuropathy. Chemotherapy-induced peripheral neuropathy (CIPN) is characterized by mechanical allodynia, paralysis, pain, tingling, and numbness, with a characteristic "stocking-andglove" distribution of symptoms [1]. CIPN is one of the dose-limiting side effects in cancer chemotherapy and may persist even after the discontinuation of drug therapy. Even worse, CIPN leads to an impairment in patients' quality of life (QOL) due to its effects on mobility and simple daily activities [2]. CIPN symptoms are difficult to manage using currently available therapeutic drugs. Furthermore, the underlying mechanism of CIPN is still not fully understood. Therefore, the discovery of new therapeutic agents to treat CIPN is an area of high priority.

Goshajinkigan in Japanese (GJG; 牛車腎気丸, Ji Sheng Shen Qi Wan in Chinese) [3], a Kampo (Japanese traditional medicine) formula composed of ten crude drugs [Rehmanniae Radix (地黄), Achyranthis Radix (牛膝), Corni Fructus (山茱莫), Dioscoreae Rhizoma (山薬), 
Plantaginis Semen (車前子), Alismatis Rhizoma (沢瀉), Poria (获苓), Moutan Cortex (牡丹皮), Cinnamomi Cortex (桂皮), and Processi Aconiti Radix (附子)], has traditionally been used for the treatment of motor weakness of the lower back and legs, sensitivity to cold, pain, numbness, reduced micturition, nocturnal enuresis, edema, and lumbago [4], in addition to symptoms of peripheral neuropathy. Based on empirical evidence of its efficacy, GJG has been used to treat diabetic neuropathy [5, 6], and the clinical use of GJG for the treatment and prevention of CIPN is increasing [7, 8]. Although several studies have examined the effects of GJG on CIPN [8,9], the active constituents of GJG responsible for efficacy in CIPN and the molecular mechanism of their pharmacological effect are not fully understood.

Previously, we compared the anti-allodynic effect of GJG and Hachimijiogan, a related formulation, using a PTX induced allodynia mouse model. Hachimijiogan is derived from GJG and lacks two crude drug ingredients, Plantaginis Semen and Achyranthis Radix. Interestingly, while GJG exhibited anti-allodynic effects, Hachimijiogan was devoid of this activity. These observations suggest that Plantaginis Semen and/or Achyranthis Radix may be involved in the anti-allodynic activity of GJG [10]. Follow-up study revealed that the administration of water extract of Plantaginis Semen (EPS, $0.3 \mathrm{~g} / \mathrm{kg}$, oral, daily) resulted in significant inhibition of PTX-induced mechanical allodynia. This effect of EPS was dose-dependent as a lower dose of EPS (0.1 g/ $\mathrm{kg}$, oral, daily) lacked significant anti-allodynic activity. In contrast, the administration of the water extract of Achyranthis Radix (EAR, 0.3-0.03 g/kg, oral, daily) did not inhibit the PTX-induced mechanical allodynia [11], suggesting the presence of active chemical constituents in Plantaginis Semen with anti-allodynic properties. In the present study, identification of anti-allodynic compounds in EPS using activity-guided separation of EPS and the evaluation of isolated compounds in PTX-induced allodynia mouse model was performed.

\section{Materials and methods}

\section{Samples and isolation}

The water extract of Plantaginis Semen (the seed of Plantago asiatica) was provided by Tsumura \& Co (Lot No. 1049). The extract $(170 \mathrm{~g})$ was first dissolved in $8.5 \mathrm{~L}$ of water followed by addition of $12.75 \mathrm{~L}$ of methanol $(\mathrm{MeOH})$ to make a $60 \% \mathrm{MeOH}$ solution, which resulted in precipitation of polysaccharide sediment. After centrifugation at $4000 \mathrm{rpm}$ for $15 \mathrm{~min}$, supernatant was separated from sediment. The supernatant was diluted to $5 \% \mathrm{MeOH}$ solution by addition of water and was subjected to Diaion HP-21 (Mitsubishi Chemical, Tokyo, Japan) column chromatography
(100 mm i.d. $\times 600 \mathrm{~mm}$, stepwise gradient elution with $0-100 \% \mathrm{MeOH}$ ) and six fractions were eluted: water eluent (3650 mg), 20\% MeOH eluent (1099 mg), 40\% MeOH eluent $(2853 \mathrm{mg}$ ), $60 \% \mathrm{MeOH}$ eluent (4800 mg), $80 \% \mathrm{MeOH}$ eluent $(852 \mathrm{mg})$, and $100 \% \mathrm{MeOH}$ eluent $(397 \mathrm{mg})$. With the help of Nippon Funmatsu Yakuhin Co., LTD (Osaka, Japan), scale-upped extraction from $8.1 \mathrm{~kg}$ of Plantaginis Semen and removal of polysaccharide sediment as described above yielded $380 \mathrm{~g}$ of processed extract (Lot No. 15D20). A portion $(160 \mathrm{~g})$ of the processed extract was subjected to Diaion HP-21 column chromatography as described above to yield $10.4 \mathrm{~g}$ of $20 \% \mathrm{MeOH}$ eluent.

Octadecylsilyl medium-pressure liquid chromatography (ODS-MPLC) was performed (Biotage SNAP Ultra C18 $120 \mathrm{~g}, 42 \mathrm{~mm}$ i.d. $\times 150 \mathrm{~mm}$ ) on $8.8 \mathrm{~g}$ the of $20 \% \mathrm{MeOH}$ fraction followed by elution with a gradient of $5-100 \%$ $\mathrm{MeOH}$ in water, to obtain seven fractions, $1 \mathrm{~A}$ to $1 \mathrm{G}$. Fraction $1 \mathrm{C}(1.39 \mathrm{~g})$ was further separated by preparative $\mathrm{C} 30$ HPLC [Develosil C30-UG-5, $20 \mathrm{~mm}$ i.d. $\times 250 \mathrm{~mm}$ with $20 \mathrm{~mm}$ i.d. $\times 50 \mathrm{~mm}$ guard column; flow rate $10 \mathrm{~mL} / \mathrm{min}$; solvent A: water with $0.1 \%$ formic acid; solvent $\mathrm{B}: \mathrm{CH}_{3} \mathrm{CN}$; gradient elution $4 \%$ solvent $\mathrm{B}(0-10 \mathrm{~min})$ and $4-30 \%$ solvent B (10-32 min), detection: UV at $210 \mathrm{~nm}$ ] which resulted in isolation of compounds $4\left(23.5 \mathrm{mg}, \mathrm{t}_{R} 12 \mathrm{~min}\right)$, 3 (13.8 mg, $\left.\mathrm{t}_{R} 16.5 \mathrm{~min}\right), \mathbf{1}\left(19.6 \mathrm{mg}, \mathrm{t}_{R} 20 \mathrm{~min}\right)$, and $\mathbf{2}$ $\left(74.2 \mathrm{mg}, \mathrm{t}_{R} 24.5 \mathrm{~min}\right)$. Tap water was used for the extraction and HP-21 column chromatography, while purified water was used for the MPLC and preparative HPLC. These isolated compounds were identified by ${ }^{1} \mathrm{H}-\mathrm{NMR}$ (ECA-500II spectrometer, JEOL, Tokyo, Japan) and high-resolution electrospray ionization time-of-flight mass spectrometry (HRESI-TOFMS, Shimadzu, Kyoto, Japan). For compounds 1 and $\mathbf{2}$, commercially available standards were also used for identification by LCMS.

In order to prepare the samples for animal experiments, the above mentioned methods using Diaion HP-21 column chromatography and ODS-MPLC were employed with slight modifications to obtain the fractions crude 3 and crude 4 . The content of $\mathbf{3}$ or $\mathbf{4}$ in crude $\mathbf{3}$ and crude $\mathbf{4}$ was estimated by quantitative ${ }^{1} \mathrm{H}$-NMR method (see Supplementary material). For preparation for $\mathbf{2}$, in addition to the above mentioned methods, preparative C30 HPLC was employed with slight modifications.

\section{Animal experiments}

\section{Animals}

Six-weeks old male C57BL/6NCr mice (Japan SLC, Shizuoka, Japan) were used in the mouse model of allodynia. Mice were housed under controlled temperature $\left(21-23^{\circ} \mathrm{C}\right)$, humidity (45-65\%), and light (7:00 AM to 7:00 PM, 12-h light/dark cycle) conditions. Food and water were available 
ad libitum. This study was approved by the Committee for Animal Experiments at the University of Toyama and was performed in accordance with the guidelines for investigations of experimental pain in animals published by the International Association for the Study of Pain.

\section{Drugs}

PTX (Sigma, St. Louis, MO, USA) was dissolved in the vehicle [vehicle 1; physiological saline containing $10 \%$ Cremophor $\mathrm{EL}^{\circledR}$ (Sigma) and 10\% ethanol] and was administered intraperitoneally $(5 \mathrm{mg} / \mathrm{kg})$ at $0.1 \mathrm{~mL} / 10 \mathrm{~g}$ of body weight. The dose of PTX was calculated based on the recommended clinical doses [12]. The fractions, purified compounds, and aucubin (Wako Pure Chemical Industries, Osaka, Japan) were dissolved in 5\% solution of gum arabic in water (vehicle 2) and administered orally in a volume of $0.1 \mathrm{~mL} / 10 \mathrm{~g}$ of body weight once daily, starting the day after PTX injection.

\section{Behavioral experiments}

Mechanical allodynia of the hind paw was assessed using a fine von Frey filament with a bending force of $0.69 \mathrm{mN}$ (North Coast Medical Inc., Morgan Hill, CA, USA) [12]. The mice were placed individually in an acrylic cage $(11 \mathrm{~cm} \times 18 \mathrm{~cm} \times 15 \mathrm{~cm})$ with a wire mesh bottom. After an acclimation period of at least $30 \mathrm{~min}$, the von Frey filament was pressed perpendicularly against the central part of the plantar hind paw of a freely-moving mouse and was held there for 1-3 s with the filament slightly buckled. Responses to the stimulus were ranked as follows: 0 , no reaction; 1 , lifting of the hind paw; and 2, licking and flinching of the hind paw. The stimulation of the same intensity was applied to each hind paw three times at intervals of several seconds, and the allodynia score (the total score of six tests) was expressed as a percentage of a maximum score of 12 .

\section{Statistical analysis}

Data were represented as the mean \pm standard error of the mean (SEM). Statistical significance was determined using two-way repeated measures analysis of variance (ANOVA) and Bonferroni post hoc test for multiple comparisons. A $p$ value of less than 0.05 was considered statistically significant.

\section{Results}

In order to identify the anti-allodynic compounds in Plantaginis Semen, activity-guided separation of its hot water extract was conducted. After removal of polysaccharide sediment by precipitation using $\mathrm{MeOH}$, the supernatant was subjected to Diaion HP-21 column chromatography to obtain six fractions (water eluent, $20 \%, 40 \%, 60 \%, 80 \%$, and $100 \%$ $\mathrm{MeOH}$ eluent). The anti-allodynic effects of these fractions were evaluated in PTX-treated mice. A single administration of PTX ( $5 \mathrm{mg} / \mathrm{kg}$, i.p.) induced mechanical allodynia. In this model, the allodynia score peaked on day 14 and almost renormalized by day 39 [11]. Therefore, we evaluated the effect of fractions and compounds on allodynia each day up to day 14 after PTX injection. The allodynia score for all fractions was the same as the vehicle 2 group, except for the $20 \% \mathrm{MeOH}$ eluent $(0.3 \mathrm{~g} / \mathrm{kg})$, which decreased the allodynia score significantly (Fig. 1). These results suggested the presence and enrichment of anti-allodynic compounds in $20 \% \mathrm{MeOH}$ eluent fraction. Scale-up of the extraction process allowed ODS-MPLC separation of the $20 \% \mathrm{MeOH}$ fraction to yield seven subfractions, $1 \mathrm{~A}-1 \mathrm{G}$. Among these seven fractions, only fraction $1 \mathrm{C}(0.15 \mathrm{~g} / \mathrm{kg})$ showed antiallodynic activity (Fig. 2). The allodynia score for fractions $1 \mathrm{E}-1 \mathrm{G}$ showed no significant difference from the vehicle 2 group (data not shown). From fraction 1C, four compounds (1-4) were purified by preparative C30 HPLC. These compounds were identified to be aucubin (1) [13], geniposidic acid (2) [14], pedicularis-lactone (3) [15], and iridolactone (4) [15], by means of spectroscopic analysis and by comparison of their MS and NMR data with those in the published literature (Fig. 3). To evaluate the anti-allodynic activity for these compounds in vivo, more than $300 \mathrm{mg}$ of the samples were prepared as follows. Commercially available $\mathbf{1}$ was used, while 2 was purified using preparative C30 HPLC. Due to their low content in Plantaginis Semen, preparation

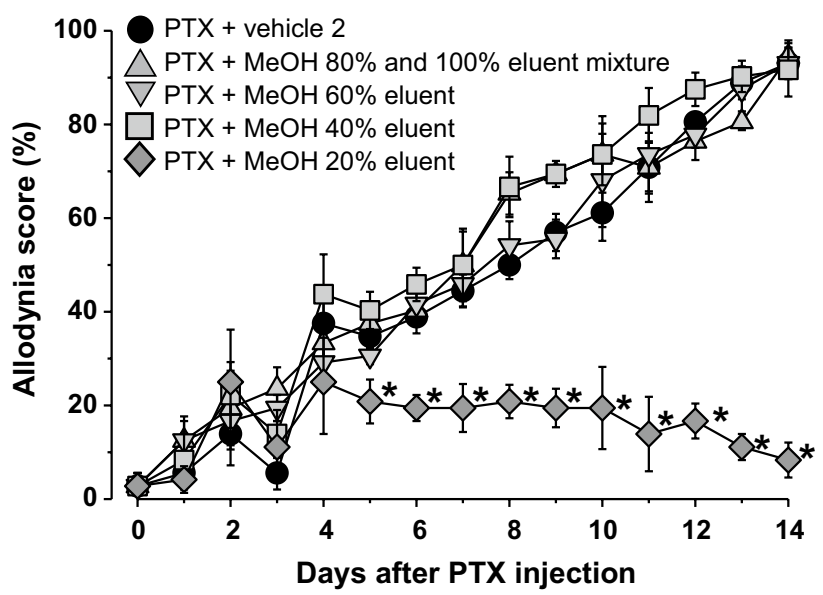

Fig. 1 Effects of repetitive oral administration of the fractions separated by Diaion HP-21 column chromatography on PTX-induced mechanical allodynia. PTX $(5 \mathrm{mg} / \mathrm{kg})$ was injected intraperitoneally in mice. Fractions or vehicle 2 (5\% gum arabic in water) were administered orally once daily starting the day after PTX injection. Data are presented as mean \pm standard error of the mean $(N=6) .{ }^{*} p<0.05$ vs. $\mathrm{PTX}+$ vehicle 2 (Bonferroni multiple comparisons) 


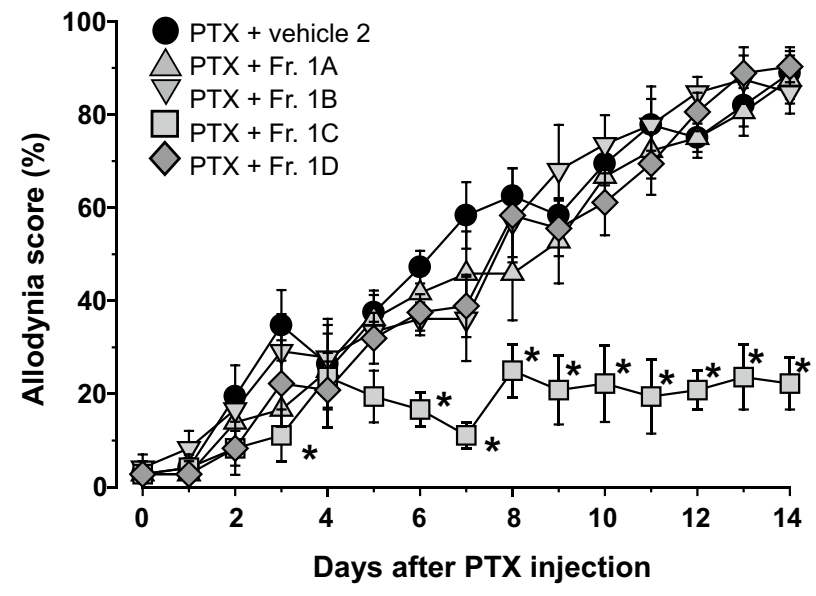

Fig. 2 Effects of repetitive oral administration of the fractions (Fr. 1A-1D) separated by MPLC on PTX-induced mechanical allodynia. PTX $(5 \mathrm{mg} / \mathrm{kg})$ was injected intraperitoneally in mice. Fractions or vehicle 2 (5\% gum arabic in water) were administered orally once daily starting the day after PTX injection. Data are presented as mean \pm standard error of the mean $(N=6)$. $* p<0.05$ vs. PTX + vehicle 2 (Bonferroni multiple comparisons)

of purified $\mathbf{3}$ and $\mathbf{4}$ for animal experiments was challenging. However, fractions crude 3 and crude 4, containing these compounds, respectively, were prepared by ODS-MPLC and used for activity evaluation in mice. Using ${ }^{1} \mathrm{H}-\mathrm{NMR}$, we determined that the principal compound in each fraction was 3 or 4, respectively. Quantitative ${ }^{1} \mathrm{H}-\mathrm{NMR}$ showed that the estimated content of $\mathbf{3}$ in crude $\mathbf{3}$ was $13.4 \pm 0.2 \%$, and that of 4 in crude 4 was $8.4 \pm 0.7 \%$.

Anti-allodynic effect of the isolated compounds was evaluated using a PTX-induced mechanical allodynia mouse model. Daily oral administration of aucubin $(\mathbf{1}, 100 \mathrm{mg} / \mathrm{kg})$, starting the day after PTX injection, significantly inhibited mechanical allodynia, with effects observed from day 8 onwards. A lower dose of $\mathbf{1}(30 \mathrm{mg} / \mathrm{kg})$ also retained the similar inhibition, evident after day 8 (Fig. 4a). Geniposidic acid $(2,75 \mathrm{mg} / \mathrm{kg})$, as shown in Fig. 4b, significantly

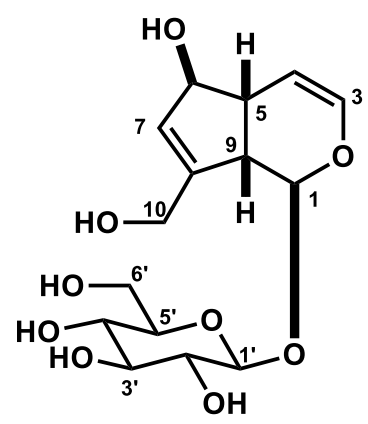

aucubin (1)

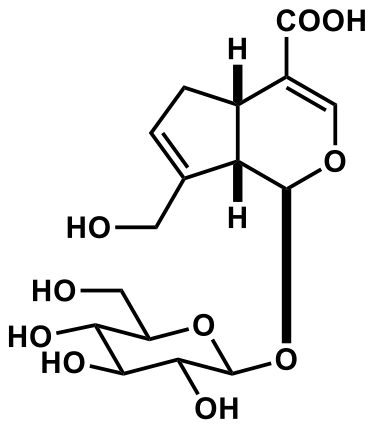

geniposidic acid (2)

decreased the allodynia score on days 7,9, and 10 compared to the vehicle 2 treated group. However, this effect was not sustained, and the score gradually increased in a manner similar to vehicle control, suggesting that $\mathbf{2}$ did not affect the mechanical allodynia induced by PTX. As mentioned above, the activities of pedicularis-lactone (3) and iridolactone (4) were evaluated using a fraction containing $\mathbf{3}$ as its major component (named as crude 3 ) and a fraction containing 4 as its major component (crude 4), respectively. Significant inhibition of mechanical allodynia was observed starting at day 6 upon oral administration of crude $3(100 \mathrm{mg} / \mathrm{kg})$, an effect also observed for a lower dose of crude $3(30 \mathrm{mg} / \mathrm{kg})$, although statistically insignificant (Fig. 5a). Oral administration of crude 4 (30 and $100 \mathrm{mg} / \mathrm{kg}$ ) exhibited no inhibition (Fig. 5b).

\section{Discussion}

Previously we have reported that once daily oral administration of GJG $(1.0 \mathrm{~g} / \mathrm{kg})$ [10] and EPS $(0.3 \mathrm{~g} / \mathrm{kg})$ [11], an ingredient crude drug of GJG, inhibit PTX-induced mechanical allodynia in mice. These findings suggest that the antiallodynic activity of GJG could be ascribed to Plantaginis Semen. In the present study, therefore, activity-guided separation of EPS was performed and led to identification of four iridoids (1-4). Aucubin (1) and geniposidic acid (2) have previously been known to be the major compounds in Plantaginis Semen [16]. The iridoids, pedicularis-lactone (3) and iridolactone (4), have been isolated from the roots of Pedicularis chinensis [15] (Oribanchaceae), the roots of Scrophularia buergeriana [17] (Scrophulariaceae), and the fruits of Vitex rotundifolia [18] (Lamiaceae). Recently, their isolation from Plantaginis Semen has been reported [19].

Oral administration of 1 (30 and $100 \mathrm{mg} / \mathrm{kg}$ ) inhibited mechanical allodynia in the PTX-induced mouse model of allodynia. Due to challenges in isolation of pure compounds sufficient for animal experiments, crude 3 and crude 4 were

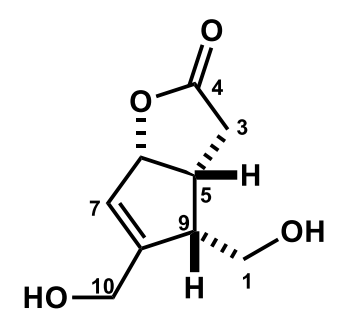

pedicularis-lactone (3)<smiles>O=C1C[C@@H]2[C@@H](C(CO)=C[C@@H]2O)[C@H](I)O1</smiles>

iridolactone (4)

Fig. 3 Structure of compounds 1-4 

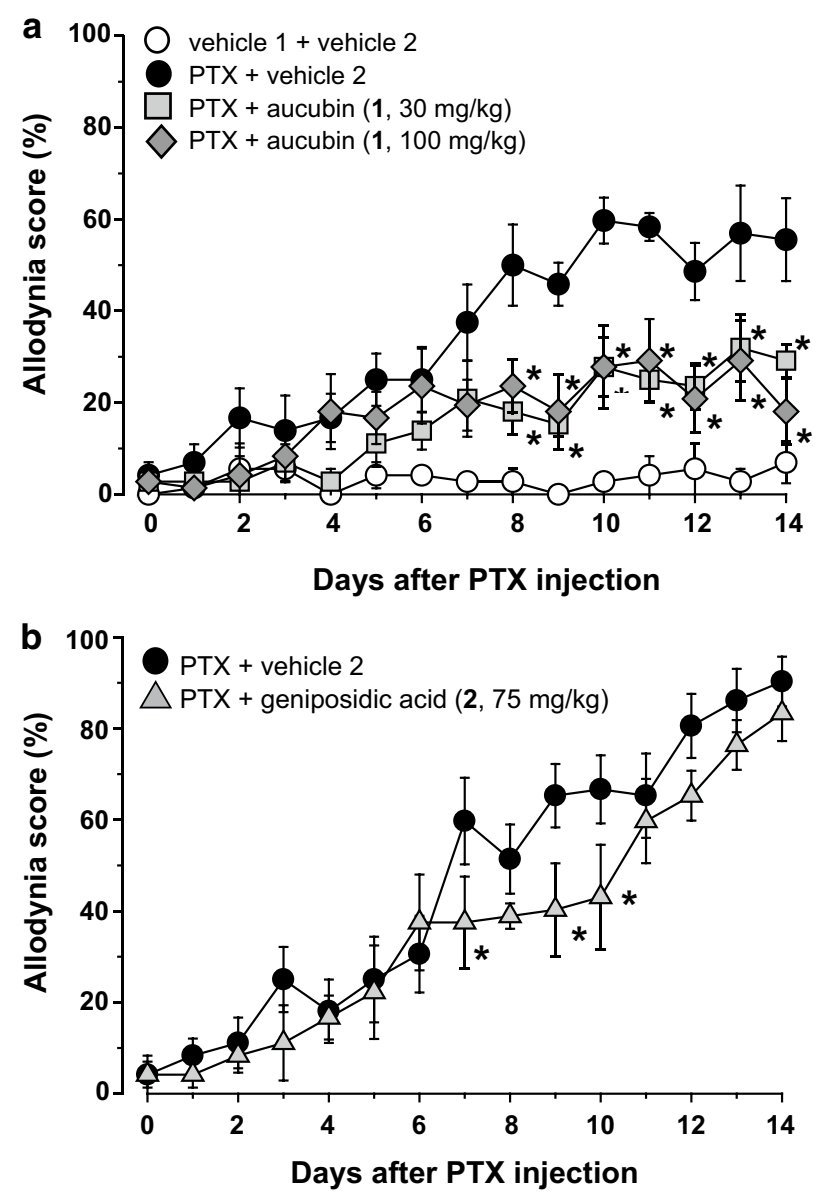

Fig. 4 Effects of repetitive oral administration of aucubin (1) and geniposidic acid (2) on PTX-induced mechanical allodynia. PTX $(5 \mathrm{mg} / \mathrm{kg}$ ) or vehicle 1 (physiological saline containing 10\% Cremophor $\mathrm{EL}^{\circledR}$ (Sigma) and 10\% ethanol) was injected intraperitoneally in mice. Aucubin $(\mathbf{1}, \mathbf{a})$, geniposidic acid $(\mathbf{2}, \mathbf{b})$, or vehicle $2(5 \%$ gum arabic in water) was administered orally once daily from the day after PTX injection. Data are presented as mean \pm standard error of the mean $(N=6)$. ${ }^{*} p<0.05$ vs. PTX + vehicle 2 (Bonferroni multiple comparisons)

evaluated in lieu of pure compounds $\mathbf{3}$ and $\mathbf{4}$. Orally administered crude $3(100 \mathrm{mg} / \mathrm{kg})$ inhibited mechanical allodynia, suggesting the potential anti-allodynic activity of $\mathbf{3}$. On the other hand, $\mathbf{2}$ and crude $\mathbf{4}$ did not affect the mechanical allodynia. Collectively, these results indicate $\mathbf{1}$ and $\mathbf{3}$ as the active anti-allodynic compounds in Plantaginis Semen and GJG. Although the effects of Plantaginis Semen extract on pain perception haven't yet been reported, the analgesic effect of oral administration of iridoids, including $\mathbf{1}$ and $\mathbf{2}$, was reported in a mouse model [20]. Thus, it is plausible that the analgesic activity of 1 may have a role in its antiallodynic effect.

Although the quantitative levels of $\mathbf{3}$ in crude $\mathbf{3}$ was not high, it is the major compound of crude 3 as suggested by ${ }^{1} \mathrm{H}-\mathrm{NMR}$ spectral data. Since tap water was used in the
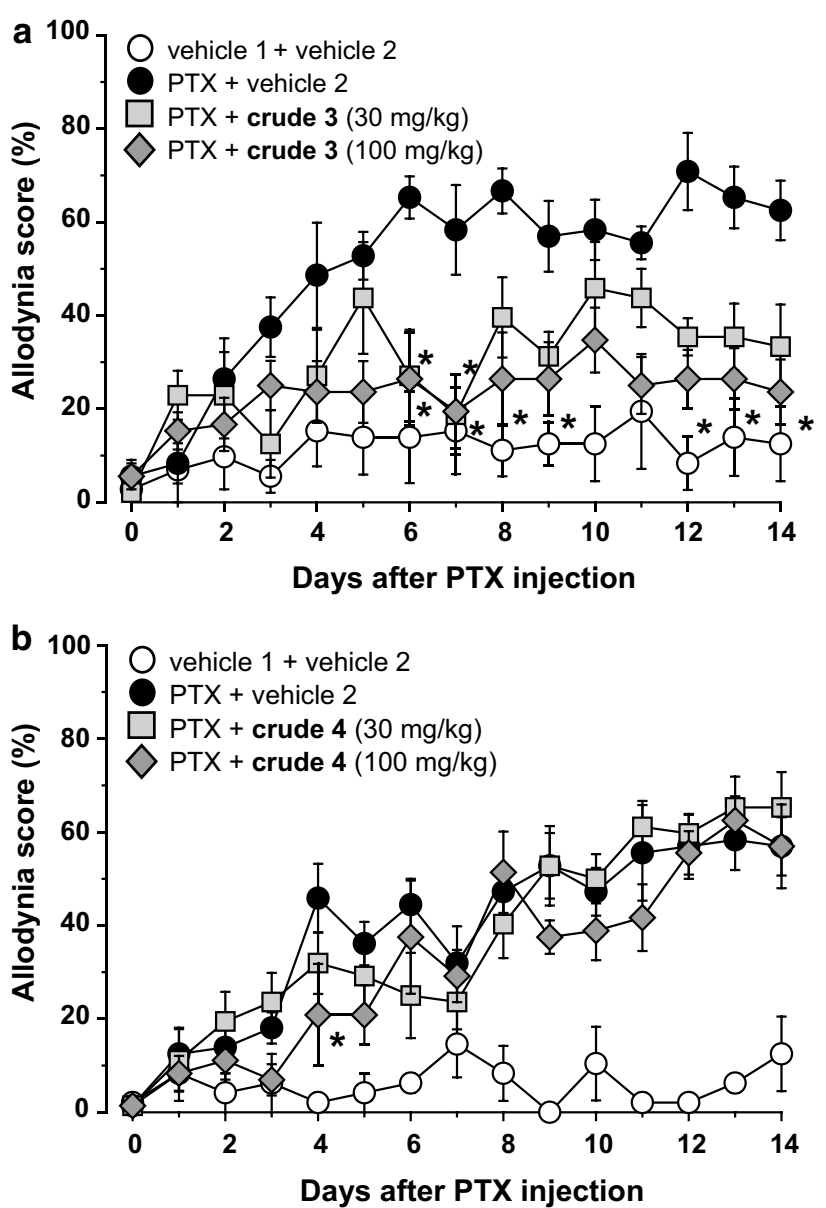

Fig. 5 Effects of repetitive oral administration of crude 3 or crude 4 on PTX-induced mechanical allodynia. PTX $(5 \mathrm{mg} / \mathrm{kg})$ or vehicle 1 (physiological saline containing 10\% Cremophor EL ${ }^{\circledR}$ (Sigma) and $10 \%$ ethanol) was injected intraperitoneally in mice. Crude 3 (a), crude 4 (b) or vehicle 2 (5\% gum arabic) were administered orally once daily starting the day after PTX injection. Data are presented as mean \pm standard error of the mean $(N=4-6)$. $* p<0.05$ vs. PTX + vehicle 2 (Bonferroni multiple comparisons)

isolation process, it is likely that other constituents of crude $\mathbf{3}$ are water soluble compounds such as minerals and sugars. Minerals are undetectable in the ${ }^{1} \mathrm{H}-\mathrm{NMR}$ as they lack a proton in the molecule. ${ }^{1} \mathrm{H}-\mathrm{NMR}$ signals, likely originating from sugars, ranging between 3 and 4 ppm were detected. Based on our current knowledge of crude 3 constituents, we postulate that 3 is one of the active anti-allodynic compounds in Plantaginis Semen and GJG. Evaluation of purified compound $\mathbf{3}$ in animal experiments will be needed to obtain additional confirmation. The purification of $\mathbf{3}$ from Plantaginis Semen presented significant challenges due to its low levels. Therefore, the purification of $\mathbf{3}$ from other crude drugs is in currently being pursued.

Xue et al. report the pharmacokinetics of aucubin intravenous administration as follows: plasma aucubin level reaches peak concentration about $0.08 \mathrm{~h}$ after administration 
(Tmax) and the elimination half-life $\left(T_{1 / 2}\right)$ is $1.07 \mathrm{~h}$. And also, they indicate that pharmacokinetic property of aucubin in TCM extract may change and may be inhibited depending on the other ingredients [21]. Regarding oral administration of aucubin, Xu et al. report that Tmax was about $1 \mathrm{~h}$ and $T_{1 / 2}$ is $7.4 \mathrm{~h}$ [22]. In our mouse experiment, evaluation of allodynia (von Frey test) was conducted after approximately $24 \mathrm{~h}$ of administration. The plasma level of aucubin may not be high at the time point of the von Frey test. As shown in Ref. [11], Andoh et al. report that single intraperitoneal injection of aucubin does not inhibit PTX-induced mechanical allodynia, suggesting that aucubin does not have acute anti-allodynic activity. Taken together, it is suggested that the alteration of plasma level of aucubin and inhibition of PTX-induced allodynia do not have direct correlation. Antiallodynic activity by repetitive administration of aucubin may be due to organic protection, such as the protection of demyelination [23].

In our previous study [11], we have reported that repetitive intraperitoneal injection of aucubin $(\mathbf{1}, 50 \mathrm{mg} / \mathrm{kg})$ inhibits mechanical allodynia induced by PTX in mice. Additionally, intraperitoneally administered $2(50 \mathrm{mg} / \mathrm{kg})$ and catalpol $(50 \mathrm{mg} / \mathrm{kg})$, related iridoid glucosides of aucubin, did not inhibit allodynia in mice. In this study, we validated the anti-allodynic effect of $\mathbf{1}$ by oral administration. In our preliminary experiment, we detected $\mathbf{1}$ in the plasma sample of mice after oral administration of EPS $(1 \mathrm{~g} / \mathrm{kg})$, suggesting that orally administered $\mathbf{1}$ was absorbed into the blood to exhibit anti-allodynic effects (data not shown). In a study on a mouse model of breast cancer, repetitive oral administration of GJG $(1 \mathrm{~g} / \mathrm{kg})$ inhibited the PTX-induced allodynia without affecting the anti-cancer activity of PTX [24]. We speculate that $\mathbf{1}$ and $\mathbf{3}$, the active anti-allodynic constituents of Plantaginis Semen will not interfere with chemotherapy. However, further studies are needed to confirm this assumption.

There is evidence to suggest the underlying mechanism of anti-allodynic activity of $\mathbf{1}$. We reported that intraperitoneal injection of $\mathbf{1}$ inhibited firing induced by mechanical stimuli in spinal dorsal horn neurons in PTX-treated mice. Furthermore, an endoplasmic reticulum (ER) stress inhibition mechanism is suggested by the inhibitory effect of $\mathbf{1}$ on the PTX-induced expression of CCAAT/enhancer-binding protein homologous protein, a protein marker of ER stress, in the mouse sciatic nerve and LY-PPB6 cells (a rat Schwann cell line) [23]. Demyelination associated with chronic pain is induced by PTX in peripheral nerves $[25,26]$, because PTX causes damage in the Schwann cells [25], which form myelin sheaths, via ER stress [27, 28]. As mentioned above, we detected aucubin in the plasma sample of mice after oral administration of EPS. Therefore, we expect that oral administration of $\mathbf{1}$ may produce anti-allodynic effects via the same mechanism as intraperitoneal administration.
Recently case reports of association between the use of Kampo formulas, especially containing Gardeniae Fructus (GF), and mesenteric phlebosclerosis are increasing [29]. Among several iridoid glycosides reported from GF, the responsible compound for MP in GF is suggested to be geniposide [30, 31]. On the other hand, although several iridoid glycosides from Plantaginis semen had been reported, to the best of our knowledge, the isolation of geniposide from Plantaginis Semen (the seed of Plantago asiatica) had not been reported $[32,33]$. The contents of major iridoid glycosides in Plantaginis Semen had been reported as follows: catalpol, $179.6 \mu \mathrm{g} / \mathrm{g}$; aucubin, $104.2 \mu \mathrm{g} / \mathrm{g}$; and geniposidic acid, $7552 \mu \mathrm{g} / \mathrm{g}$ [34]. The Japanese Pharmacopoeia, Seventeenth Edition (JP17) prescribes that GF contains not less than $3.0 \%$ of geniposide. Thus, the content of iridoid glycosides in Plantaginis Semen could be considered to be lower than the geniposide content in GF.

Normally the period of anti-cancer agent treatment is less than one year, in cancer chemotherapy. Consequently, the period of GJG treatment for CIPN is also less than one year. According to Nagata's report [29], the average period of GF administration in MP case patients was 11.1 years (4.0-15.9 years). In our 14-day mice experiment the darkpurple change of mucosal surface in the colon, a typical finding of MP, was not observed when administered the fraction or compound. In the present, there is not enough information on MP and long period administration of GJG. Iridoid glycoside content is low in GJG, however, the long period of administration should be noted on the adverse effects, including MP.

\section{Conclusion}

Our study helps to clarify the active constituents of GJG and its ingredient crude drug, Plantaginis Semen, which possess anti-allodynic activity. Activity-guided separation of the hot water extract of Plantaginis Semen led to the isolation of four iridoids (1-4). In addition to aucubin (1), repetitive oral administration of crude pedicularis-lactone (3) showed anti-allodynic action. Collectively, this study suggest that $\mathbf{1}$ and $\mathbf{3}$ can be some of the active anti-allodynic compounds in Plantaginis Semen and GJG. Aucubin (1) and pedicularislactone (3) have the potential for therapeutic application in the clinical management of CIPN.

Acknowledgement This study was supported in part by a Grant-inAid for the Cooperative Research Project from Institute of Natural Medicine, University of Toyama in 2016 and 2017, JSPS KAKENHI Grant Number 15K07993 and 18K06728, Wakanyaku-Biotechnology Research Grant from Toyama prefecture, and JSPS Core-to-Core Program, Asia-Africa Science Platforms. The authors would like to thank Tsumura \& Co. and Nippon funmatsu yakuhin Co., LTD, for providing the samples. 


\section{Compliance with ethical standards}

Conflict of interest The authors declare that they are no conflict of interest.

Open Access This article is licensed under a Creative Commons Attribution 4.0 International License, which permits use, sharing, adaptation, distribution and reproduction in any medium or format, as long as you give appropriate credit to the original author(s) and the source, provide a link to the Creative Commons licence, and indicate if changes were made. The images or other third party material in this article are included in the article's Creative Commons licence, unless indicated otherwise in a credit line to the material. If material is not included in the article's Creative Commons licence and your intended use is not permitted by statutory regulation or exceeds the permitted use, you will need to obtain permission directly from the copyright holder. To view a copy of this licence, visit http://creativecommons.org/licenses/by/4.0/.

\section{References}

1. Han Y, Smith M (2013) Pathobiology of cancer chemotherapyinduced peripheral neuropathy (CIPN). Front Pharmacol 4:156. https://doi.org/10.3389/fphar.2013.00156

2. Wolf S, Barton D, Kottschade L, Grothey A, Loprinzi C (2008) Chemotherapy-induced peripheral neuropathy: prevention and treatment strategies. Eur J Cancer 44:1507-1515. https://doi. org/10.1016/j.ejca.2008.04.018

3. Department of Pharmacognosy, Phytochemistry and Narcotics (DPPN), National Institute of Health Sciences (NIHS) of Japan and National Institutes of Biomedical Innovation, Health and Nutrition (NIBIOHN). Standards of Reporting Kampo Products (STORK) (http://mpdb.nibiohn.go.jp/stork/)

4. Institute of Natural Medicine, University of Toyama. Traditional Medical \& Pharmaceutical Database (http://dentomed.toyam a-wakan.net/en/medical_information_on_kampo_formulas/gosha jinkigan/SCP000052)

5. Tawata M, Kurihara A, Nitta K, Iwase E, Gan N, Onaya T (1994) The effects of Goshajinkigan, a herbal medicine, on subjective symptoms and vibratory threshold in patients with diabetic neuropathy. Diabetes Res Clin Pract 26:121-128

6. Watanabe K, Shimada A, Miyaki K, Hirakata A, Matsuoka K, Omae K, Takei I (2014) Long-term effects of goshajinkigan in prevention of diabetic complications: a randomized open-labeled clinical trial. Evid Based Complement Altern Med 2014:128726. https://doi.org/10.1155/2014/128726

7. Yamamoto T, Murai T, Ueda M, Katsuura M, Oishi M, Miwa Y, Okamoto Y, Uejima E, Taguchi T, Noguchi S, Kurokawa N (2009) Clinical features of paclitaxel-induced peripheral neuropathy and role of Gosya-jinki-gan. Gan To Kagaku Ryoho 36:89-92 (Article in Japanese)

8. Cascella M, Muzio MR (2017) Potential application of the Kampo medicine goshajinkigan for prevention of chemotherapyinduced peripheral neuropathy. J Integr Med 15:77-87. https:// doi.org/10.1016/S2095-4964(17)60313-3

9. Suzuki T, Miyamoto K, Yokoyama N, Sugi M, Kagioka A, Kitao Y, Adachi T, Ohsawa M, Mizukami H, Makino T (2016) Processed aconite root and its active ingredient neoline may alleviate oxaliplatin-induced peripheral neuropathic pain. J Ethnopharmacol 186:44-52. https://doi.org/10.1016/j.jep.2016.03.056

10. Andoh T, Kitamura R, Kuraishi Y, Fushimi H, Komatsu K, Shibahara N (2014) Effects of goshajinkigan, hachimijiogan, and rokumigan on mechanical allodynia induced by Paclitaxel in mice. J Tradit Complement Med 4:293-297. https://doi. org/10.4103/2225-4110.128906

11. Andoh T, Kato M, Kitamura R, Mizoguchi S, Uta D, Toume K, Komatsu K, Kuraishi Y (2016) Prophylactic administration of an extract from Plantaginis Semen and its major component aucubin inhibits mechanical allodynia caused by paclitaxel in mice. J Tradit Complement Med 6:305-308. https://doi.org/10.1016/j.jtcme .2015.12.001

12. Gauchan P, Andoh T, Kato A, Sasaki A, Kuraishi Y (2009) Effects of the prostaglandin $\mathrm{E}_{1}$ analog limaprost on mechanical allodynia caused by chemotherapeutic agents in mice. J Pharmacol Sci 109:469-472

13. Davini E, Iavarone C, Trogolo C, Aureli P, Pasolini B (1986) The quantitative isolation and antimicrobial activity of the aglycon of aucubin. Phytochemistry 25:2420-2422

14. Toda S, Miyase T, Arichi H, Tanizawa H, Takino Y (1985) Natural antioxidants. II. Antioxidative components isolated from seeds of Plantago asiatica Linne. Chem Pharm Bull 33:1270-1273

15. Li Y, Changzeng W, Zhongjian J (1995) Iridoids in roots of Pedicularis chinensis. Phytochemistry 40:491-494

16. Kawamura T, Hisata Y, Okuda K, Hoshino S, Noro Y, Tanaka T, Kodama A, Nishibe S (1998) Pharmacognostical Study of Plantaginis Herba (13) Constituents of Seeds of Plantago sp. and Commercial Plantago Seeds. Nat Med 52:5-9

17. Lin SJ, Tan CH, Jiang SH, Li YM, Zhu DY (2006) C9-iridoids from Scrophularia buergeriana. Helv Chim Acta 89:2789-27893. https://doi.org/10.1002/hlca.200690250

18. Ono M, Ito Y, Kubo S, Nohara T (1997) Two new iridoids from Viticis trifoliae Fructus (fruit of Vitex rotundifolia L.). Chem Pharm Bull 45:1094-1096. https://doi.org/10.1248/cpb.45.1094

19. Peng QH, Han ZZ, Tong RC, Hu XQ, Yang QM, Qi M, Shi YH, Wang ZT, Yang L (2017) Chemical constituents from hypoglycemic active part of Plantaginis Semen. Zhongguo Zhong Yao Za Zhi 42:4150-4153. https://doi.org/10.19540/j.cnki.cjcmm.20170 905.009 (Article in Chinese)

20. Okuyama E, Fujimori S, Yamazaki M, Deyama T (1998) Pharmacologically active components of Viticis Fructus (Vitex rotundifolia) II The component having analgesic Effects. Chem Pharm Bull 46:655-662. https://doi.org/10.1248/cpb.46.655

21. Xue B, Ma B, Zhang Q, Li X, Zhu J, Liu M, Wu X, Wang C, Wu $Z$ (2015) Pharmacokinetics and tissue distribution of Aucubin, Ajugol and Catalpol in rats using a validated simultaneous LCESI-MS/MS assay. J Chromatogr B 1002:245-253. https://doi. org/10.1016/j.jchromb.2015.08.026

22. Xu W, Deng Z, Guo H, Ling P (2012) A rapid and sensitive determination of aucubin in rat plasma by liquid chromatography-tandem mass spectrometry and its pharmacokinetic application. Biomed Chromatogr 26:1066-1070. https://doi.org/10.1002/ bmc. 1748

23. Andoh T, Uta D, Kato M, Toume K, Komatsu K, Kuraishi Y (2017) Prophylactic administration of aucubin inhibits paclitaxelinduced mechanical allodynia via the inhibition of endoplasmic reticulum stress in peripheral schwann cells. Biol Pharm Bull 40:473-478. https://doi.org/10.1248/bpb.b16-00899

24. Bahar MA, Andoh T, Ogura K, Hayakawa Y, Saiki I, Kuraishi Y (2013) Herbal medicine goshajinkigan prevents paclitaxel-induced mechanical allodynia without impairing antitumor activity of paclitaxel. Evid Based Complement Altern Med 2013:849754. https://doi.org/10.1155/2013/849754

25. Cavaletti G, Tredici G, Braga M, Tazzari S (1995) Experimental peripheral neuropathy induced in adult rats by repeated intraperitoneal administration of taxol. Exp Neurol 133:64-72. https://doi. org/10.1006/exnr.1995.1008 
26. Komiya Y (1992) Changes of fast axonal transport by taxol injected subepineurally into the rat sciatic nerve. Neurosci Res 14:159-165. https://doi.org/10.1016/0168-0102(92)90077-P

27. Liao P-C, Tan S-K, Lieu C-H, Jung H-K (2008) Involvement of endoplasmic reticulum in paclitaxel-induced apoptosis. J Cell Biochem 104:1509-1523. https://doi.org/10.1002/jcb.21730

28. Tanimukai H, Kanayama D, Omi T, Takeda M, Kudo T (2013) Paclitaxel induces neurotoxicity through endoplasmic reticulum stress. Biochem Biophys Res Commun 437:151-155. https://doi. org/10.1016/j.bbrc.2013.06.057

29. Nagata $Y$, Watanabe T, Nagasaka K, Yamada M, Murai M, Takeuchi S, Murase M, Yazaki T, Murase T, Komatsu K, Kaizuka M, Sano M, Asano K, Ando C, Taniuchi N (2016) Total dosage of gardenia fruit used by patients with mesenteric phlebosclerosis. BMC Complement Altern Med 16:207. https://doi.org/10.1186/ s12906-016-1182-1

30. Hiramatsu K, Sakata H, Horita Y, Orita N, Kida A, Mizukami A, Miyazawa M, Hirai S, Shimatani A, Matsuda K, Matsuda M, Ogino H, Fujinaga H, Terada I, Shimizu K, Uchiyama A, Ishizawa S, Abo H, Demachi H, Noda Y (2012) Mesenteric phlebosclerosis associated with long-term oral intake of geniposide, an ingredient of herbal medicine. Aliment Pharmacol Ther 36:575-586. https:// doi.org/10.1111/j.1365-2036.2012.05221
31. Ohtsu K, Matsui T, Nishimura T, Hirai F, Ikeda K, Iwashita A, Yorioka M, Hatakeyama S, Hoashi T, Koga Y, Sakurai T, Miyaoka M (2014) Association between mesenteric phlebosclerosis and Chinese herbal medicine intake. Nihon Shokakibyo Gakkai Zasshi 111:61-68 (Article in Japanese)

32. Shan MQ, Yu S, Yan H, Guo S, Xiao W, Wang ZZ, Zhang L, Ding AW, Wu QA, Li SFY (2017) A review on the phytochemistry, pharmacology, pharmacokinetics and toxicology of geniposide, a natural product. Molecules 22:1689. https://doi.org/10.3390/ molecules22101689

33. Zhou Q, Lu W, Niu Y, Liu J, Zhang X, Gao B, Akoh CC, Shi $\mathrm{H}, \mathrm{Yu} \mathrm{L}$ (2013) Identification and quantification of phytochemical composition and anti-inflammatory, cellular antioxidant, and radical scavenging activities of 12 Plantago species. J Agric Food Chem 61:6693-6702. https://doi.org/10.1021/jf401191q

34. Kim BH, Lee NK, Chang IM (2009) Simultaneous analysis of a mixture of iridoids in water extracts of Plantago asiatica L. by LC. Chromatographia 69:1397-1400. https://doi.org/10.1365/ s10337-009-1069-0

Publisher's Note Springer Nature remains neutral with regard to jurisdictional claims in published maps and institutional affiliations. 